\title{
ESTUDIO DE MICROFÓSILES DE RESIDUOS ADHERIDOS EN PIPAS CERÁMICAS DEL SITIO VILLA JMC-1 LABRANZA, REGIÓN DE LA ARAUCANÍA ${ }^{1}$
}

\author{
LUCIANA D. QUIROZ, CAROLINA A. BELMAR*, M. TERESA \\ PLANELLA"**, RODRIGO MERA* Y DOINA MUNITA****
}

\section{RESUMEN}

Se aplica el estudio de microfósiles en dos pipas del sitio Villa JMC-1, definido como un contexto funerario del Complejo Pitrén, con el fin de reconocer qué plantas fueron utilizadas en la acción de fumar. Si bien en las fuentes escritas hay múltiples referencias describiendo esta actividad en ocasiones rituales, no hay evidencias arqueológicas directas de cuáles plantas fueron consumidas. En esta perspectiva se propone confrontar la información etnohistórica y etnográfica con la evidencia arqueobotánica recuperada de los residuos recuperados en los hornillos y tubos/boquilla de las pipas.

Se constató la presencia diferencial de almidones y silicofitolitos en ambas partes de las pipas, exhibiendo las boquillas mayor cantidad de almidones y en los hornillos menor frecuencia y diversidad de microfósiles. Entre los taxa identificados, destacan morfotipos adscritos a Poaceae y Solanaceae, además de almidones simil Nicotiana sp. y Solanum sp.

PALABRAS CLAVES: microfósiles, residuos adheridos, pipas, complejo Pitrén.

\section{MICROFOSSIL STUDY OF CERAMIC SMOKING PIPE RESIDUES, VILLA JMC-1 LABRANZA SITE, REGION OF THE ARAUCANIA, CHILE}

\section{ABSTRACT}

The study of microfossils is applied to two pipes from the Villa JMC-1 site, site which has been defined as a funerary context from the Pitrén Complex, with the objective of recognizing which plants were used in the act of smoking. Although many written sources make reference to this activity during rituals, there is no direct archaeological evidence of which plants could have been consumed. In this perspective, this study aims to confront the ethnohistoric and ethnographic information with the archaeobotanical evidence recovered from the residues found in the chamber and tubes/mouthpieces of the pipes.

1 Proyecto FONDART "El pasado enterrado de Labranza: 1.000 años de alfarería, textilería y orfebrería en la Araucanía", Investigadores Responsables: Rodrigo Mera y Doina Munita.

* Licenciada en Arqueología, Universidad de Chile. lucianaquiroz@hotmail.com, meragol@gmail.com.

* Docente, Universidad Internacional SEK, Av. Arrieta 1000, Peñalolén. carolina_belmar@hotmail.com.

** Arqueóloga, Magíster en Historia mención Etnohistoria. Universidad de Chile. Sociedad Chilena de Arqueología. mtplanella@ gmail.com.

*s** Magíster en Planificación y Gestión Territorial, Universidad Católica de Temuco. doinamunita@yahoo.com. 
The differential presence of starches and silicophytoliths in both parts of the pipes was confirmed. The mouthpieces presented a greater amount of starches, while the chamber presented less frequency and diversity of microfossils. Among the identified taxa, two morphotypes assigned to Poaceae and Solanaceae stand out, as well as simile Nicotiana sp. and Solanum sp. starch grains.

KEYWORDS: microfossils, residues, smoking pipes, Pitrén complex.

\section{INTRODUCCIÓN}

En un importante cementerio adscrito al complejo funerario Pitrén del período Alfarero Temprano del área sur de Chile, Villa JMC-1 Labranza, sin precedentes en cuanto a su registro material (Mera y Munita 2008), se han recuperado dos pipas o cachimbas con las cuales se propuso determinar su contenido a través del análisis de microfósiles.

El estudio de los residuos adheridos a estas dos pipas constituye una vía de aproximarse en mayor medida a la comprensión de los contextos funerarios Pitrén, en consideración de su alto significado ritual y su íntimo vínculo con la expresión material de ritos sociales. Esto es confirmado por los estudios etnográficos disponibles para la zona sur en que la producción de humo y su utilización simbólica juega un rol fundamental (Guevara 1911; Föerster 1993; Föerster 1995 y Gundermann 1997)².

En esta perspectiva, previa descripción de la metodología usada, se expondrán los resultados del análisis de las muestras extraídas directamente desde el interior de hornillos y boquillas de las pipas del sitio Villa JMC-1, Labranza para luego ser discutidos.

\section{ANTECEDENTES DE LA TÉCNICA DE ESTUDIOS DE MICROFÓSILES}

Por microfósil se entiende cualquier organismo muerto, vulnerable a los procesos naturales de sedimentación y erosión, es un fósil, independiente del modo en que se preservó o tiempo transcurrido desde su muerte (Armstrong y Brasier 2005[1980]:

2 Del mismo modo, la costumbre y uso de pipas para fumar tiene un importante registro histórico para las regiones australes de Argentina y Chile, entre los Tehuelches, Fueguinos y Chonos, destacando la pipa tipo "monitor". Esta costumbre fue adquirida por influencia de grupos mapuche en el siglo XVII y XVIII (Cooper 1949, Guevara y Oyarzún 1912, Serrano 1934) de quienes adoptaron la connotación ritual del acto de fumar y el esquema particular de participación durante su desarrollo (Martinic 1991).
3). Los microfósiles pueden ser de origen animal o vegeta

Una misma planta puede producir diferentes sustancias biogénicas que se conservan como microfósiles después de su muerte. Estas sustancias pueden proceder de manera exclusiva de una parte de la planta o bien, distribuirse en diferentes órganos o partes en ella (Pearsall 1989). En virtud de esta particularidad, el estudio acabado de las microrrestos en función de su origen anatómico revela ser de utilidad para la identificación taxonómica de los microrrestos vegetales adheridos en artefactos arqueológicos, ya que demuestran además mejores índices de conservación que los macrorrestos en el mismo tiempo transcurrido. Esta línea de estudio se ha aplicado en artefactos como vasijas, implementos de molienda, instrumental lítico y piedras tacitas, permitiendo abordar preguntas relativas a la funcionalidad de los artefactos, así como de modos de procesamiento y uso de los recursos vegetales (ver Briuer 1976; Loy 1994; Fullagar et al. 1996; Barton et al. 1998; Piperno y Holst 1998; Kealhofer et al. 1999; Babot 2004; Loy y Fullagar 2006; Planella et al. 2011).

La propuesta de este análisis incluye la determinación taxonómica y anatómica de los microrrestos/microfósiles presentes en las sustancias adheridas a las pipas cerámicas del sitio Villa JMC-1, Labranza, de la novena región. Este estudio se basará en la línea del análisis múltiple de microfósiles (Coil et al. 2003, Korstanje 2009) que contempla la recuperación e identificación de la totalidad de microfósiles presentes, lo que amplia los márgenes de identificación e interpretación.

Este enfoque se complementa beneficiosamente con los estudios de modalidades de procesamiento (Babot 2003, 2006), que se valen de las huellas tafonómicas o patrones de daños básicos de los microrrestos, para refrendar una secuencia de tratamientos y procesamientos de las plantas y sus partes, cuando se combinan entre sí. Por 


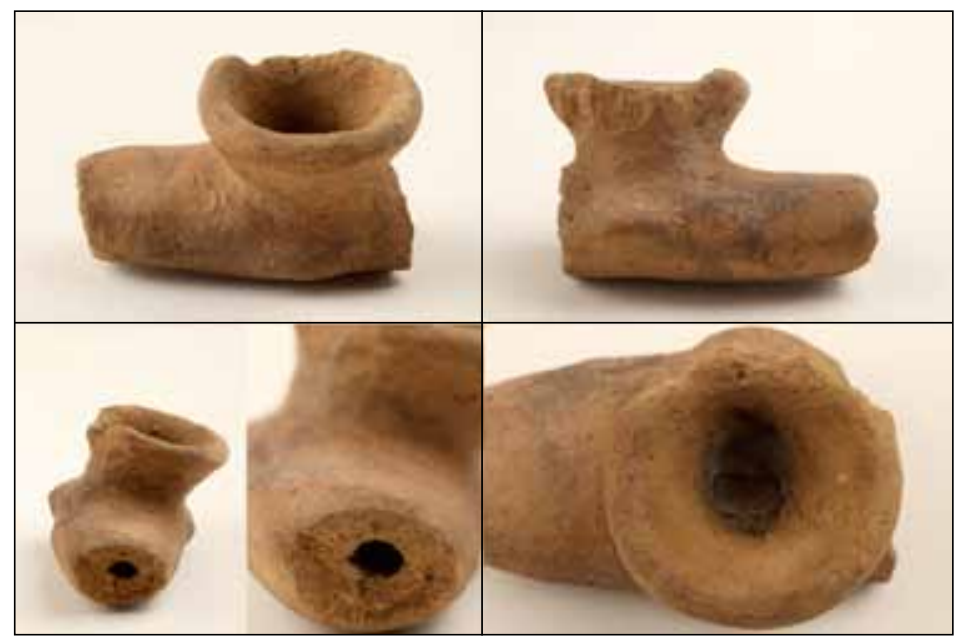

Figuras 1, 2, 3 y 4. Pipa del rasgo 4, sector casa 1, sitio Villa JMC01, Labranza, Temuco, Región de La Araucanía.

ejemplo, los almidones son particularmente sensibles a cambios de temperatura (hervido, tostado, quemado), humedad y fricción mecánica, tras los cuales se gelatinizan, fisuran, abollan, fracturan, pierden parcial o completamente sus propiedades ópticas (birrefringencia), los granos compuestos se desagregan y, por último, se perforan luego de ser fermentados (Babot 2003, 2006).

\section{MATERIALES Y MÉTODOS}

El material objeto de este estudio está compuesto por muestras de contenidos (residuos adheridos) de 2 pipas arqueológicas cerámicas del área sur (Villa JMC-1, Labranza, Temuco). Se adscriben a la Cultura Pitrén, datadas en Cal. AD 890 a 1.030 (Cal. BP 1.060 a 920 con 95\% de probabilidad; Mera y Munita 2008). La primera proviene del sector Casa 1, Rasgo $\mathrm{n}^{\circ} 4$, y la segunda fue recuperada sin contexto (ver Tabla 1).

Es importante acotar que la excavación de este sitio se efectuó mientras se edificaban los cimientos de una villa de casas, por lo tanto los contextos se encontraban en alguna medida alterados. En la Casa $\mathrm{N}^{\circ} 1$ se identificó un total de 9 rasgos. Entre ellos, el Rasgo $N^{\circ} 4$ (Figura 1) se describe como un conjunto de piezas alfareras y fragmentos cerámicos, que presentaban evidencias de intervención (Mera y Munita 2008).

La naturaleza orgánica de algunos de los microrrestos los hace más susceptibles a ser destruidos por las técnicas de extracción basadas en aplicación de reactivos ácidos (Pearsall 1989), por lo que se recurrió a una técnica menos agresiva y adecuada para piezas arqueológicas, el raspado directo (Loy 1994), que además permite cumplir a cabalidad los objetivos del análisis múltiple de microfósiles (ver Kealhofer et al. 1999; Babot 2003, 2004; Korstanje 2005, 2006, 2009). Se extrajeron muestras desde dos sectores diferentes de las pipas, hornillos y tubos/boquillas, esperando encontrar diferencias entre los resultados de ambas procedencias.

La observación de los preparados se realizó bajo microscopio petrográfico que cuenta con lente micrograduado, polarizador, analizador de cuarzo y cámara incorporada. Se trabajó con aumentos 200x y 500x.

La descripción de la morfología y atributos de los silicofitolitos se basó en el International Code for Phytolith Nomenclature 1.0 generado por el ICPN Working Group (2005) con el fin de adscribirlos a un taxon. Se utilizaron los criterios de 1) formas tridimensional o en su defecto bidimensional, 2) aspecto de la superficie, y 3) origen anatómico, cuando corresponde (tricoma o pelo, célula epidérmica). En

Tabla 1. Procedencia de las muestras extraídas en las pipas del Rasgo 4, Casa 1 y sin contexto del sitio JMC-1, Labranza, Temuco, IX ${ }^{\text {a Región. }}$

\begin{tabular}{|c|c|c|}
\hline \multirow{2}{*}{ Sitio, Pipa } & \multicolumn{2}{|c|}{ Raspado Directo } \\
\cline { 2 - 3 } & Hornillo & Tubo \\
\hline Labranza, JMC-1, Rasgo 4, Casa 1 & $\mathrm{X}$ & $\mathrm{X}$ \\
\hline Labranza, JMC-1, sin contexto & $\mathrm{X}$ & $\mathrm{X}$ \\
\hline
\end{tabular}


el caso de los almidones, descripción se anotó la presencia de rasgos diagnósticos, como lamellas, fisura o cicatrices (Babot 2004, 2007, Korstanje y Babot 2007). De igual, manera, se identificaron, midieron, describieron y anotaron los atributos relevantes de otros microfósiles visualizados en las preparaciones.

La identificación se realizó por comparación con la colección de referencia de microfósiles disponible y con el apoyo de publicaciones especializadas (Reichert 1913; Piperno 1988, 2006; Pearsall y Piperno 1993; Capperelli et al. 2006; Korstanje y Babot 2007; Planella et al. 2009; MU Phytholith Database).

\section{RESULTADOS}

\section{Pipa Sin Contexto}

Muestra Procedente de Boquilla

Los microfósiles detectados en la boquilla suman 40 ejemplares, mostrando una amplia variedad de formas y distintas procedencias anatómicas. Se observaron dos morfotipos de almidones sin determinación taxonómica, uno de forma hexagonal (5\%) y otro orbicular (2,5\%). Además se recuperaron 38 silicofitolitos, entre los que se destacan base de tricoma $(2,5 \%)$, bulliforme $(12,5 \%)$, pentagonal con muesca (simil plantas gramíneas, 2,5\%), paralelepípedo con muesca (simil Bambusoideae, 2,5\%), elongado con bordes aserrados (células epidérmicas de hojas de gramíneas, 42.5\%). Finalmente, hay formas oblongas y cuadrangulares con paredes gruesas (células epidérmicas de tallos y hojas 22,5\%) (Tabla 2).

Tabla 2. Descripción de los tipos de microfósiles detallando número, forma, dimensiones $(\mu)^{1}$ y figura correspondiente, encontrados en residuos adheridos a boquilla de pipa sin contexto, sitio Villa JMC-1 Labranza, Temuco, IX Región.

\begin{tabular}{|c|c|c|c|c|c|c|c|c|c|c|c|}
\hline $\begin{array}{c}\text { Tipo } \\
\text { microfósil }\end{array}$ & Morfotipo & $\begin{array}{c}\text { Forma } \\
\text { geométrica }\end{array}$ & $\begin{array}{c}\text { Atributos/ } \\
\text { Ornamentación }\end{array}$ & $\begin{array}{l}\text { Atributos } \\
\text { brazos de } \\
\text { almidón }\end{array}$ & Número & $\%$ & $\begin{array}{c}\text { Ancho } \\
(\mu)\end{array}$ & $\begin{array}{c}\text { Largo } \\
(\mu)\end{array}$ & $\begin{array}{c}\text { Altura } \\
a(\mu)\end{array}$ & $\begin{array}{l}\mathrm{N}^{\circ} \\
\text { Fig. }\end{array}$ & $\begin{array}{l}\text { Asignación } \\
\text { taxonómica }\end{array}$ \\
\hline \multirow[t]{15}{*}{ Fitolito } & & & & & 37 & 92,5 & & & & & \\
\hline & Célula corta & & & & 14 & 35 & & & & & \\
\hline & & cúbico & & & 4 & 10,0 & 25 & 25 & & & \\
\hline & & Trapezoide & & & 3 & 7,5 & 6 & 7 & & & \\
\hline & & Paralelepípedo & muesca & & 1 & 2,5 & 7 & 4 & & 12 & Bambusoideae \\
\hline & & Pentagonal & muesca & & 2 & 5,0 & 25 & 25 & & & Poaceae \\
\hline & & Bilobulado & paredes gruesas & & 1 & 2,5 & 6 & 7 & & & \\
\hline & & Cuadrangular & paredes gruesas & & 2 & 5,0 & 6 & 7 & & & \\
\hline & & Globular & & & 1 & 2,5 & $\begin{array}{c}15 \\
\text { (diámetro) }\end{array}$ & & & & \\
\hline & Célula larga & & & & 17 & 42,5 & & & & & \\
\hline & & Elongado & $\begin{array}{c}\text { Bordes aserrados } \\
\text { o verrucados }\end{array}$ & & 17 & 42,5 & 6 & 19 & & 10 & Poaceae \\
\hline & $\begin{array}{c}\text { Célula } \\
\text { bulliforme }\end{array}$ & & & & 5 & 12.5 & & & & & \\
\hline & & Cuneiforme & & & 5 & 12.5 & 14 & 20 & & 9 & \\
\hline & Tricoma & & & & 1 & 2,5 & & & & & \\
\hline & & Acicular & & & 1 & 2,5 & 18 & 24 & & 11 & \\
\hline \multirow[t]{3}{*}{ Almidón } & & & & & 3 & 7,5 & & & & & \\
\hline & & Hexagonal & $\begin{array}{l}\text { Cruz de extinción } \\
\text { excéntrica }\end{array}$ & $\begin{array}{l}\text { Brazos } \\
\text { curvos }\end{array}$ & 2 & 5,0 & $\begin{array}{c}5 \\
\text { (diámetro) }\end{array}$ & & & 13 y 14 & \\
\hline & & Orbicular & $\begin{array}{c}\text { Cruz de extinción } \\
\text { excéntrica }\end{array}$ & $\begin{array}{l}\text { Un brazo } \\
\text { curvo y } \\
\text { otro recto }\end{array}$ & 1 & 2,5 & $\begin{array}{c}12,5 \\
\text { (diámetro) }\end{array}$ & & & 15 y 16 & \\
\hline \multicolumn{5}{|c|}{ Total microfósiles } & 40 & $100 \%$ & & & & & \\
\hline
\end{tabular}

Se han especificado las dimensiones de cada microrresto a modo de referencia, porque no han sido todos identificados taxonómicamente. Por la falta de adscripción taxonómica estos datos no fueron tratado estadísticamente. 


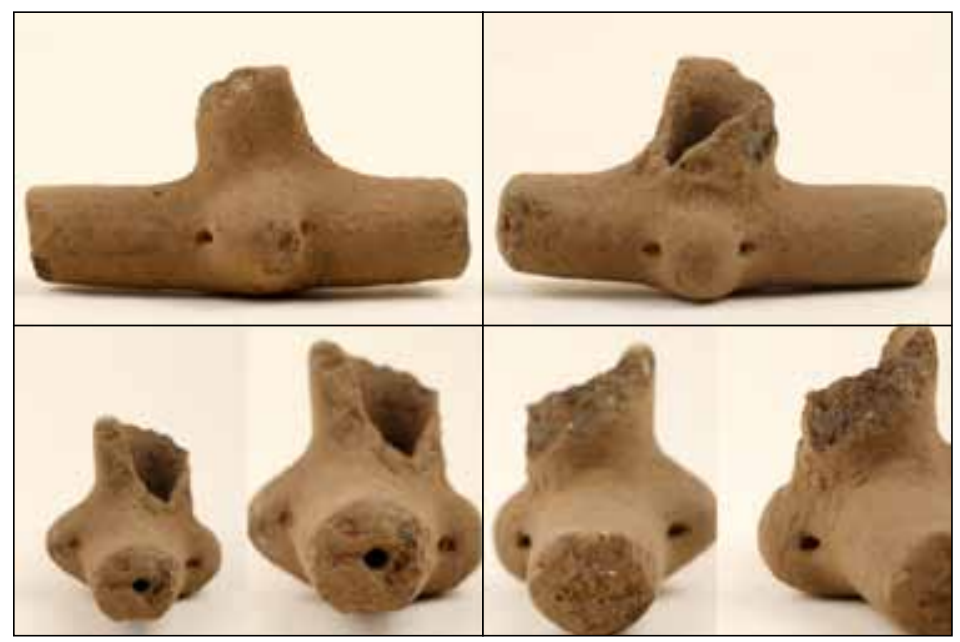

Figuras 5, 6, 7 y 8. Pipa sin contexto sitio Villa JMC01, Labranza, Temuco, Región de La Araucanía.

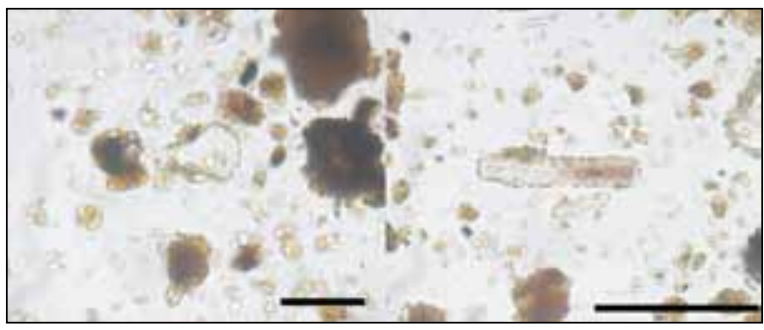

Figs. 9 y 10. Izquierda: Silicofitolito bulliforme. Derecha: Silicofitolito elongado con bordes aserrados.

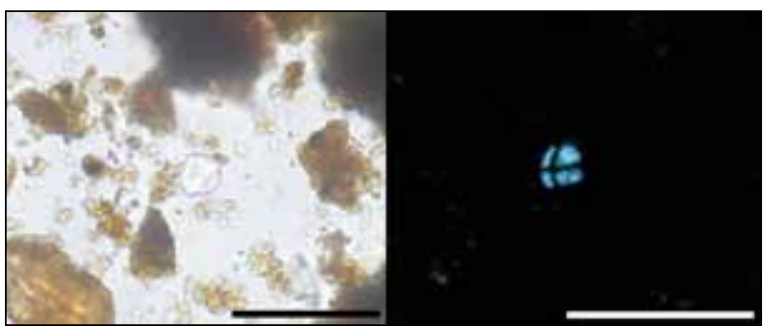

Figs. 13 y 14. Izquierda: Almidón con luz trasmitida. Derecha: mismo almidón con luz polarizada.

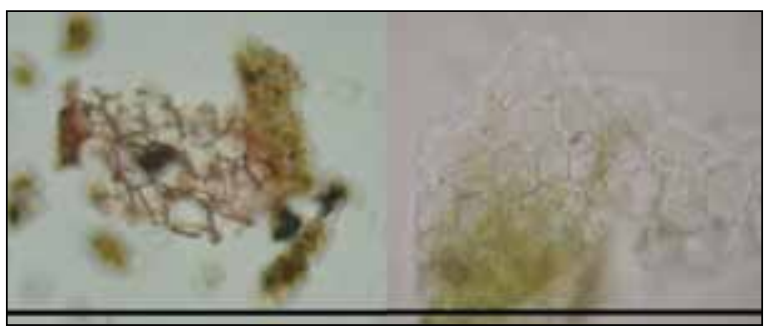

Figuras 17 y 18 . Izquierda: Silicofitolito conformado por un conjunto de células parenquimáticas. Derecha:

Conjunto células parenquimáticas registradas en Nicotiana sp. (Planella et al. 2009).

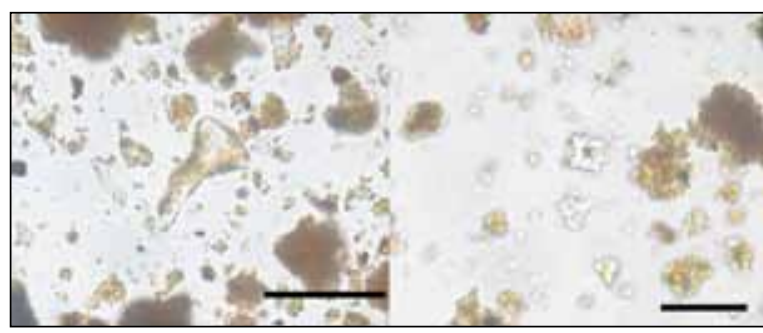

Figs. 11 y 12 . Izquierda: Silicofitolito tricoma con bordes aserrados. Derecha: Silicofitolito paralelepípedo con muesca simil Bambusoidea.

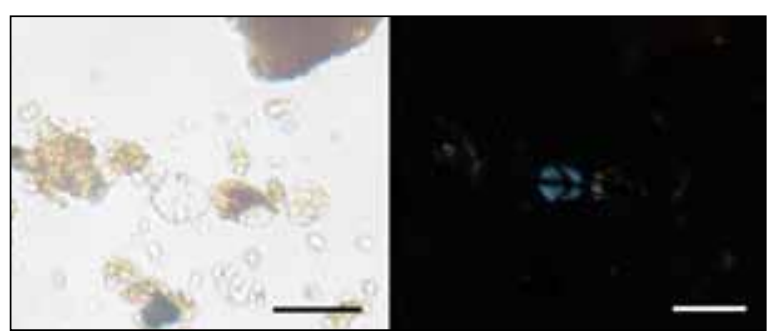

Figs. 15 y 16. Izquierda: Almidón con luz trasmitida. Se observa daño: alteración del volumen, ejemplar chato. Derecha: mismo almidón con luz polarizada.

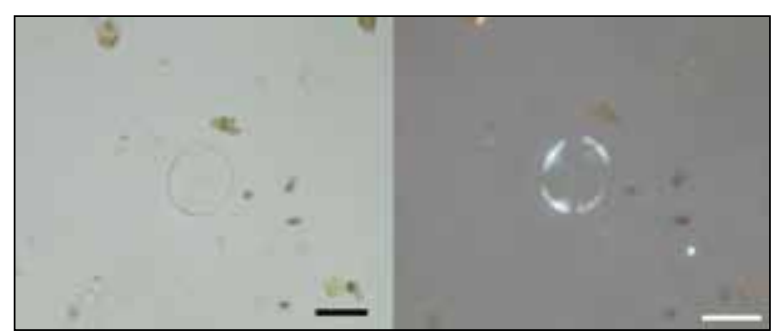

Figuras 19 y 20. Izquierda: Almidón, con luz trasmitida. Se observa daño en birrefringencia. Derecha: mismo almidón con luz polarizada. 


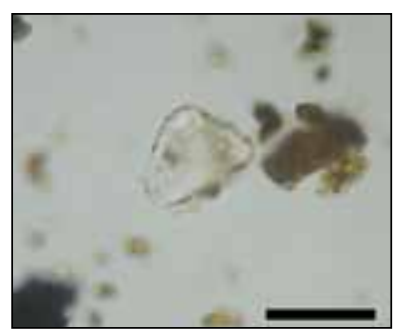

Figura 21. Silicofitolito triangular con bordes gruesos.

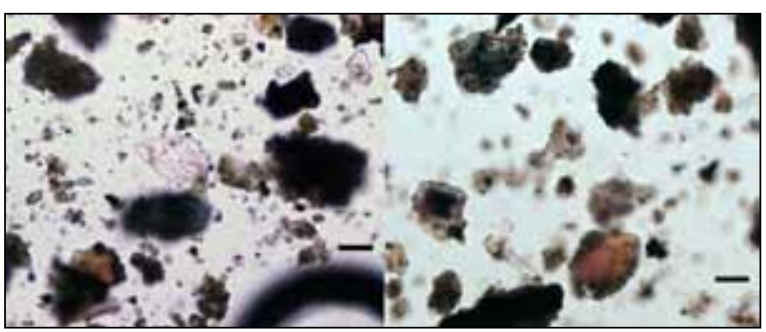

Figuras 24 y 25. Izquierda: Silicofitolito trapezoidal. Derecha: Silicofitolito tricoma de base pentagonal.

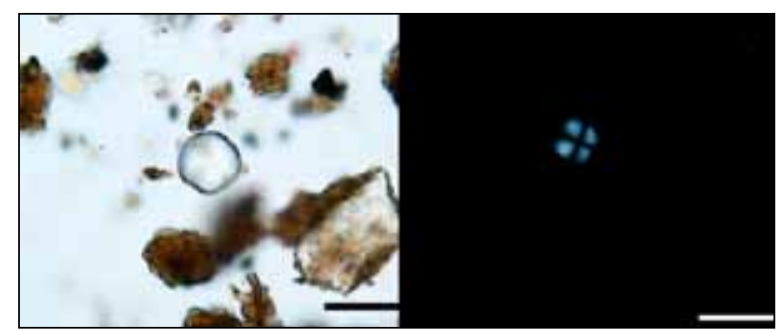

Figura 27 y 28. Izquierda: Almidón con luz trasmitida. Derecha: mismo almidón con luz polarizada, símil Nicotiana sp.

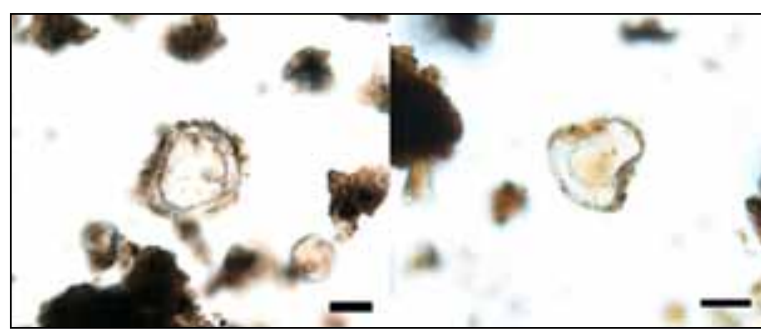

Figuras 22 y 23. Izquierda: Silicofitolito aovado con bordes gruesos. Derecha: Silicofitolito bulliforme con núcleo oscuro.

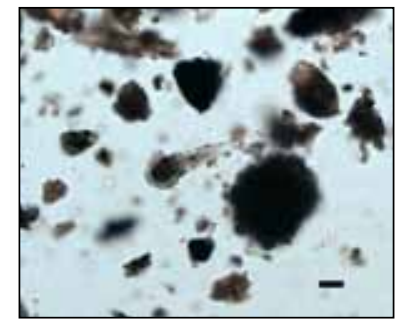

Figura 26. Izquierda: Silicofitolito tricoma de base pentagonal.

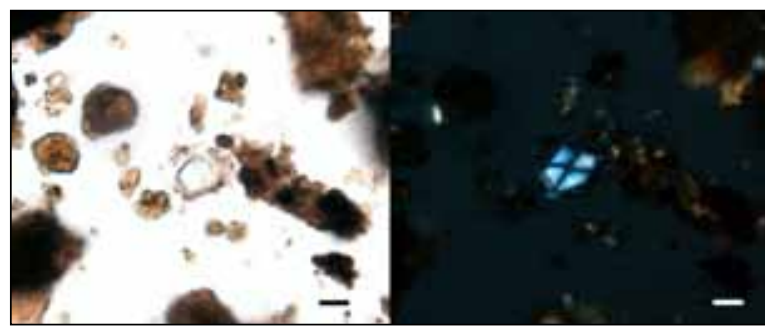

Figuras 29 y 30. Izquierda: Almidón con luz trasmitida.

Se observa daño: abolladura, alteración del hilo y de la birrefringencia. Derecha: mismo almidón con luz polarizada.

Tabla 3. Descripción de los tipos de microfósiles detallando número, forma, dimensiones $(\mu)^{1}$ y figura correspondiente, encontrados en residuos adheridos en hornillo de pipa sin contexto, sitio Villa JMC-1 Labranza, Temuco, IX Región.

\begin{tabular}{|c|c|c|c|c|c|c|c|c|c|c|c|}
\hline $\begin{array}{l}\text { Tipo } \\
\text { microfósil }\end{array}$ & Morfotipo & $\begin{array}{c}\text { Forma } \\
\text { geométrica }\end{array}$ & $\begin{array}{l}\text { Atributos/ } \\
\text { Ornamentación }\end{array}$ & $\begin{array}{l}\text { Atributos } \\
\text { brazos de } \\
\text { almidón }\end{array}$ & Número & $\%$ & $\begin{array}{l}\text { Ancho } \\
(\mu)\end{array}$ & $\begin{array}{l}\text { Largo } \\
(\mu)\end{array}$ & $\begin{array}{c}\text { Altura } \\
a(\mu)\end{array}$ & $\begin{array}{l}\mathrm{N}^{\circ} \\
\text { Fig. }\end{array}$ & $\begin{array}{l}\text { Asignación } \\
\text { taxonómica }\end{array}$ \\
\hline \multirow[t]{8}{*}{ Fitolito } & & & & & 5 & 83,33 & & & & & \\
\hline & Célula corta & & & & 4 & 66,66 & & & & & \\
\hline & & cuadrangular & $\begin{array}{c}\text { muesca y núcleo } \\
\text { oscuro }\end{array}$ & & 1 & 16,67 & 5 & 5 & & & $\begin{array}{c}\text { simil } \\
\text { Bambusoideae }\end{array}$ \\
\hline & & Trapezoide & & & 2 & 33,33 & 5 & 6 & & & \\
\hline & & triangular & paredes gruesas & & 1 & 16,67 & 15 & 17 & & 21 & \\
\hline & Célula larga & & & & 1 & 16,67 & & & & & \\
\hline & & Elongado & $\begin{array}{c}\text { Bordes aserrados } \\
\text { o verrucados }\end{array}$ & & 1 & 16,67 & 2 & 38 & & 10 & Poaceae \\
\hline & & polihédrico & psilado & & conjunto & & 7 & 10 & & 17 & \\
\hline \multirow[t]{2}{*}{ Almidón } & & & & & 1 & 16,67 & & & & & \\
\hline & & Ovalado & \begin{tabular}{|c|} 
cruz de extincíon \\
e hilo invisibles
\end{tabular} & $\begin{array}{c}\text { pérdida de } \\
\text { birrefringencia }\end{array}$ & 1 & 16,67 & 14 & 41 & & 19 y 20 & \\
\hline
\end{tabular}


Muestra Procedente de Hornillo

En el hornillo se cuentan 6 morfotipos de microfósiles entre las que se destacan un conjunto de células (tejido parenquimático), silicofitolito trapezoidal (33,33\%), silicofitolito cuadrangular con muescas (Gramínea, simil Bambusoideae, 16,65\%), almidón con pérdida de birrefringencia (16,67\%), silicofitolito triangular con paredes gruesas (célula epidérmica, $16,67 \%$ ) y silicofitolito elongado con bordes aserrados (célula epidérmica de gramínea 16,67\%) (Tabla 3).

\section{Pipa Rasgo 4, Sector Casa 1}

\section{Muestra Procedente de Boquilla}

En la boquilla se registraron 18 morfotipos. Los más frecuentes han sido los silicofitolito bulliforme con núcleo oscuro (asimilable a gramíneas, $24,24 \%)$, silicofitolito bulliforme (22,73\%), silicofitolito acicular (tricoma, epidermis de hoja o tallo, 7,58\%), silicofitolito aovado con paredes gruesas (células epidérmicas, 7,58\%), almidones $(6,06 \%$, dos de los

Tabla 4. Descripción de los tipos de microfósiles detallando número, forma, dimensiones $(\mu)^{1}$ y figura correspondiente, encontrados en residuos adheridos de boquilla de la pipa del Rasgo 4, sector Casa 1, sitio Villa JMC-1 Labranza, Temuco, IX Región.

\begin{tabular}{|c|c|c|c|c|c|c|c|c|c|c|c|}
\hline $\begin{array}{l}\text { Tipo } \\
\text { microfósil }\end{array}$ & Morfotipo & $\begin{array}{c}\text { Forma } \\
\text { geométrica }\end{array}$ & $\begin{array}{c}\text { Atributos/ } \\
\text { Ornamentación }\end{array}$ & $\begin{array}{l}\text { Atributos } \\
\text { brazos de } \\
\text { almidón }\end{array}$ & Número & $\%$ & $\begin{array}{c}\text { Ancho } \\
(\mu)\end{array}$ & $\begin{array}{c}\text { Largo } \\
(\mu)\end{array}$ & $\begin{array}{c}\text { Altura } \\
a(\mu)\end{array}$ & $\begin{array}{l}\mathrm{N}^{\circ} \\
\text { Fig. }\end{array}$ & $\begin{array}{l}\text { Asignación } \\
\text { Taxonomica }\end{array}$ \\
\hline \multicolumn{12}{|l|}{ Fitolito } \\
\hline & Célula corta & & & & 26 & 38,39 & & & & & \\
\hline & & Aovado & Paredes gruesas & & 5 & 7.58 & $38 / 28$ & 44 & & 22 & \\
\hline & & Cuadrangular & Núcleo visible & & 2 & 3.03 & 30 & 30 & & & \\
\hline & & & Liso & & 1 & 1.52 & 25 & 30 & & & \\
\hline & & Esfera & Socavada & & 1 & 1.52 & $\begin{array}{c}35 \\
\text { (diámetro) }\end{array}$ & & & & \\
\hline & & Paralelepípedo & Muesca & & 3 & 4.54 & 50 & 60 & & & Poaceae \\
\hline & & Pentagonal & Núcleo visible & & 3 & 4.54 & $30 / 42$ & $30 / 46$ & & & \\
\hline & & Rectangular & Liso & & 8 & 12.12 & $15 / 20$ & $105 / 75$ & & & \\
\hline & & Romboidal & Núcleo visible & & 1 & 1.52 & 20 & 25 & & & \\
\hline & & Trapezoide & & & 2 & 3.03 & 25 & 75 & 25 & 24 & \\
\hline & $\begin{array}{c}\text { Célula } \\
\text { bulliforme }\end{array}$ & & & & 31 & 46,96 & & & & & \\
\hline & & Cuneiforme & & & 15 & 22.73 & 40 & 60 & & & \\
\hline & & Cuneiforme & Núcleo oscuro & & 16 & 24.24 & 50 & 48 & 26 & 23 & Poaceae \\
\hline & Tricoma & & & & 5 & 7,58 & & & & & \\
\hline & & Acicular & & & 1 & 1.25 & 25 & 75 & & 26 & \\
\hline & & & $\begin{array}{l}\text { Base pentagonal } \\
\text { y núcleo visible }\end{array}$ & & 4 & 6.06 & 35 & 55 & & 25 & \\
\hline \multirow[t]{5}{*}{ Almidón } & & & & & 4 & 6,06 & & & & & \\
\hline & & Hexagonal & $\begin{array}{c}\text { Cruz de extinción } \\
\text { centrada }\end{array}$ & $\begin{array}{l}\text { Brazos rectos } \\
\text { e hilo ancho, } \\
\text { redondeado }\end{array}$ & 1 & 1.52 & 15 & 12 & & 29 y 30 & \\
\hline & & & $\begin{array}{c}\text { Cruz de extinción } \\
\text { excentrica } \\
\text { y lobulos } \\
\text { asimetricos }\end{array}$ & $\begin{array}{l}\text { Hilo visible } \\
\text { y perforado }\end{array}$ & 1 & 1.52 & 12 & 15 & 33 y 34 & & \\
\hline & & Redondeado & $\begin{array}{c}\text { Cruz de extincion } \\
\text { centrada }\end{array}$ & $\begin{array}{c}\text { Brazos rectos } \\
\text { y lobulos } \\
\text { simetricos }\end{array}$ & 1 & 1.52 & $\begin{array}{c}16 \\
\text { (diametro) }\end{array}$ & & & 27 y 28 & $\begin{array}{c}\text { símil } \\
\text { Nicotiana sp. }\end{array}$ \\
\hline & & Oblongo & $\begin{array}{l}\text { Cruz levemente } \\
\text { excentrico }\end{array}$ & $\begin{array}{c}\text { Brazos rectos } \\
\text { y delgados, } \\
\text { uno de ellos } \\
\text { dañados }\end{array}$ & 1 & 1.52 & 18 & 29 & & 31 y 32 & \\
\hline \multicolumn{5}{|c|}{ Total microfósiles } & 66 & $100 \%$ & & & & & \\
\hline
\end{tabular}




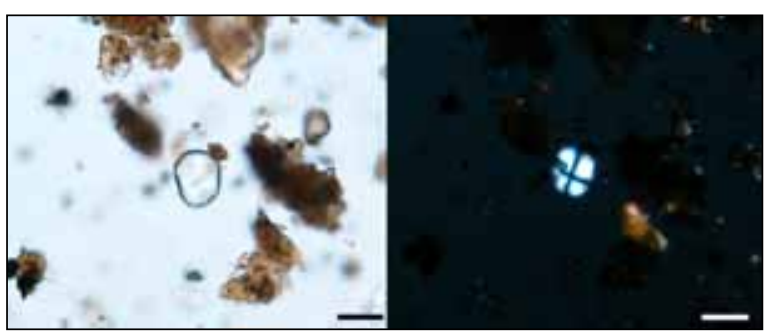

Figuras 31 y 32. Izquierda: Almidón con luz trasmitida. Se observa daño: alteración del hilo y de la cruz de extinción, fisura y abolladura.

Derecha: mismo almidón con luz polarizada.

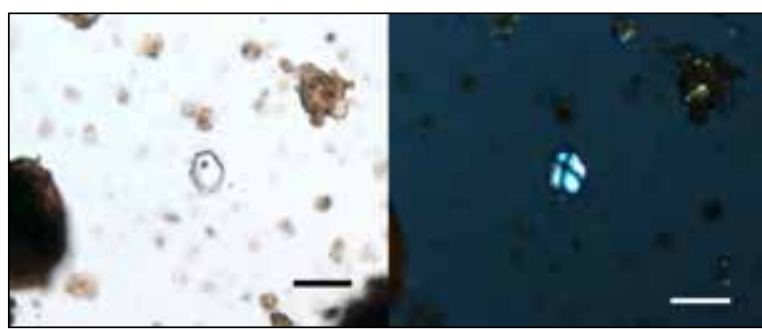

Figuras 33 y 34. Izquierda: Almidón (18x29 ) con luz trasmitida. Se observa daño: alteración del hilo y de la cruz de extinción, abolladura. Derecha: mismo almidón con luz polarizada.

Tabla 5. Descripción de los tipos de microfósiles detallando número, forma, dimensiones ( $\mu)^{1}$ y figura correspondiente, encontrados en residuos adheridos de hornillo de pipa Rasgo 4, Casa 1, sitio Villa JMC-1 Labranza, Temuco, IX ${ }^{a}$ Región.

\begin{tabular}{|c|c|c|c|c|c|c|c|c|c|c|c|}
\hline Tipo microfósil & Morfotipo & $\begin{array}{l}\text { Forma } \\
\text { geométrica }\end{array}$ & $\begin{array}{c}\text { Atributos/ } \\
\text { Ornamentación }\end{array}$ & $\begin{array}{l}\text { Atributos } \\
\text { brazos de } \\
\text { almidón }\end{array}$ & Número & $\%$ & $\begin{array}{c}\text { Ancho } \\
(\mu)\end{array}$ & $\begin{array}{c}\text { Largo } \\
(\mu)\end{array}$ & $\begin{array}{c}\text { Altura } \\
\mathrm{a}(\mu)\end{array}$ & $\begin{array}{l}\mathrm{N}^{\circ} \\
\text { Fig. }\end{array}$ & $\begin{array}{l}\text { Asignación } \\
\text { Taxonomica }\end{array}$ \\
\hline \multicolumn{12}{|l|}{ Fitolito } \\
\hline & Célula corta & & & & 26 & 38,39 & & & & & \\
\hline & & Aovado & Paredes gruesas & & 5 & 7.58 & $38 / 28$ & 44 & & 22 & \\
\hline & & Cuadrangular & Núcleo visible & & 2 & 3.03 & 30 & 30 & & & \\
\hline & & & Liso & & 1 & 1.52 & 25 & 30 & & & \\
\hline & & Esfera & Socavada & & 1 & 1.52 & $\begin{array}{c}35 \\
\text { (diámetro) }\end{array}$ & & & & \\
\hline & & Paralelepípedo & Muesca & & 3 & 4.54 & 50 & 60 & & & Poaceae \\
\hline & & Pentagonal & Núcleo visible & & 3 & 4.54 & $30 / 42$ & $30 / 46$ & & & \\
\hline & & Rectangular & Liso & & 8 & 12.12 & $15 / 20$ & $105 / 75$ & & & \\
\hline & & Romboidal & Núcleo visible & & 1 & 1.52 & 20 & 25 & & & \\
\hline & & Trapezoide & & & 2 & 3.03 & 25 & 75 & 25 & 24 & \\
\hline & $\begin{array}{c}\text { Célula } \\
\text { bulliforme }\end{array}$ & & & & 31 & 46,96 & & & & & \\
\hline & & Cuneiforme & & & 15 & 22.73 & 40 & 60 & & & \\
\hline & & Cuneiforme & Núcleo oscuro & & 16 & 24.24 & 50 & 48 & 26 & 23 & Poaceae \\
\hline & Tricoma & & & & 5 & 7,58 & & & & & \\
\hline & & Acicular & & & 1 & 1.25 & 25 & 75 & & 26 & \\
\hline & & & $\begin{array}{c}\text { Base pentagonal y } \\
\text { núcleo visible }\end{array}$ & & 4 & 6.06 & 35 & 55 & & 25 & \\
\hline \multirow[t]{5}{*}{ Almidón } & & & & & 4 & 6,06 & & & & & \\
\hline & & Hexagonal & $\begin{array}{c}\text { Cruz de extinción } \\
\text { centrada }\end{array}$ & $\begin{array}{c}\text { Brazos rectos } \\
\text { e hilo ancho, } \\
\text { redondeado }\end{array}$ & 1 & 1.52 & 15 & 12 & & $\begin{array}{c}29 y \\
30\end{array}$ & \\
\hline & & & $\begin{array}{c}\text { Cruz de extinción } \\
\text { excentrica } \\
\text { y lobulos } \\
\text { asimetricos }\end{array}$ & $\begin{array}{c}\text { Hilo visible y } \\
\text { perforado }\end{array}$ & 1 & 1.52 & 12 & 15 & 33 y 34 & & \\
\hline & & Redondeado & $\begin{array}{c}\text { Cruz de extincion } \\
\text { centrada }\end{array}$ & $\begin{array}{c}\text { Brazos rectos } \\
\text { y lobulos } \\
\text { simetricos }\end{array}$ & 1 & 1.52 & $\begin{array}{c}16 \\
\text { (diametro) }\end{array}$ & & & $\begin{array}{c}27 y \\
28\end{array}$ & $\begin{array}{c}\text { símil } \\
\text { Nicotiana sp. }\end{array}$ \\
\hline & & Oblongo & $\begin{array}{l}\text { Cruz levemente } \\
\text { excentrico }\end{array}$ & $\begin{array}{c}\text { Brazos rectos } \\
\text { y delgados, } \\
\text { uno de ellos } \\
\text { dañados }\end{array}$ & 1 & 1.52 & 18 & 29 & & $\begin{array}{c}31 y \\
32\end{array}$ & \\
\hline $\begin{array}{c}\text { Total } \\
\text { microfósiles }\end{array}$ & & & & & 66 & $100 \%$ & & & & & \\
\hline
\end{tabular}




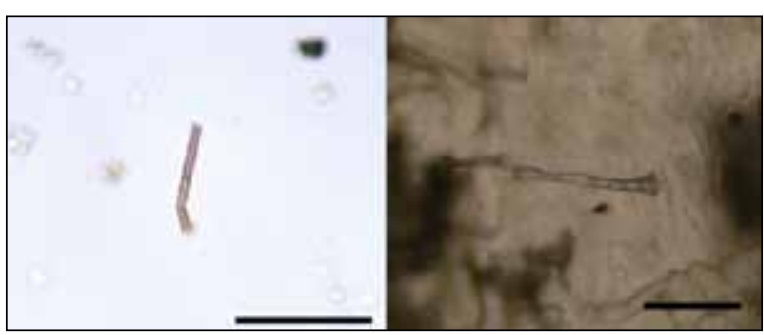

Figuras 35 y 36. Izquierda: Silicofitolito acicular articulado $(1 \times 14 \mu)$ fragmentado. Derecha:

Silicofitolito tricoma acicular articulado de Nicotiana

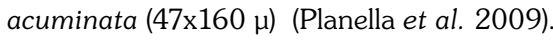

cuales presentan daños relacionado a exposición a alta temperatura o desecamiento $y$, tal vez, molienda. Uno de ellos descrito como "redondeado con cruz de extinción centrada, brazos rectos, lóbulos simétricos" se asignóó tentativamente a Nicotiana sp.), los silicofitolitos paralelepípedos con muescas (células epidérmicas de hoja de familia gramínea, 4,54\%), silicofitolito pentagonal con núcleo (base de tricoma, 4,54\%) y silicofitolito esférico con núcleo grande (no identificado, 1,52\%) (Tabla 4).

\section{Muestra Procedente de Hornillo}

En el hornillo, se registraron 6 morfotipos que corresponden a silicofitolitos elongados con bordes aserrados o verrucosos (célula epidérmica de gramínea, 46,15\%), silicofitolito orbicular (26,96\%), silicofitolito cuadrangular con paredes gruesas (epidérmica, 11,54\%) y silicofitolito cristal rectangular delgado liso (rafidio, 7,69\%), acicular, articulado (Familia de Solanaceae, 3,85\%) (Tabla 5).

\section{DISCUSIÓN}

En general es posible decir que los residuos de ambas pipas contienen silicofitolitos correspondientes a gramíneas, reconocibles a partir de la presencia de formas elongadas y aserradas, así como de paralelepípedos con núcleo visible y trapecio, típicas de células epidérmicas de hoja u otras partes aéreas de las monocotiledóneas. Algunos de los silicofitolitos paralelepípedos con núcleo, se asimilan a las formas atribuidas a la tribu Bambusoidea y en particular de Chusqueideae, en las que se incluyen la quila y el colihue, y Chusquea cumingii y Chusquea culeou, respectivamente (Piperno y Pearsall 1998, Piperno 2006).

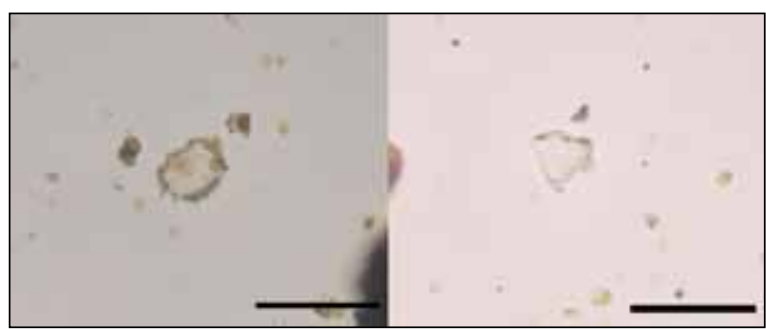

Figuras 37 y 38 . Izquierda: Silicofitolito cuadrangular con bordes gruesos $(7 \times 10 \mu)$. Derecha: Silicofitolito triangular con bordes gruesos $(7 x 8 \mu)$.

La eminente presencia de estos elementos gramíneos o pastos en el contenido de las pipas dice relación con al menos cuatro hipótesis: 1) El contenido de las pipas ha sido contaminado por la depositación natural de microfósiles en la matriz. El estudio de microfósiles de suelos y una contra-muestra de la matriz de suelo del sector de los hallazgos de las pipas, esclarecerían esta situación. 2) La familia Poaceae o gramíneas agrupan las especies más frecuentes de la cubierta herbácea y que según la literatura son las que producen silicofitolitos más abundantemente (Bertoldi de Pomar 1972; Pearsall y Piperno 1993; Zucol y Passeggi 2008). 3) En las pipas se fumaron plantas con propiedades psicoactivas junto con especies de la familia Poaceae. Uno de los mecanismos de incorporación pudo ser el uso de bosta de animales (eventualmente herbívoros consumidores de pastos) ${ }^{3}$ como catalizador de la reacción psicoactiva. 4) La presencia de estas especies responde, entre otros, al empleo de elementos del contexto de uso que incluye palos a modo de instrumento para limpiar los conductos de las pipas y los materiales usados como iniciadores de fuego. Al respecto, se menciona en la literatura el uso de colihue u otra madera adelgazada que se frotan perpendicularmente y de la presencia de hojas secas (que podrían ser de la misma especie u otra) para iniciar el fuego 4 . Es oportuno recordar aquí el procedimiento araucano de producir fuego por frotamiento [..] consistía en un palo de colihue (Chusquea coleu), como de 20 centímetros de

Guevara 1911; Guevara y Oyarzún 1912; Serrano 1934.

4 De una forma u otra, tanto las hojas de colihue como de maíz están involucradas en el acto de fumar. Joseph (1930) menciona que las hojas tiernas de estas gramíneas sirven de envoltura para los cigarrillos de tabaco. 
largo, con dos cortes superficiales paralelos, que le dan dos caras; la de abajo sirve de para que no se dé vuelta el pequeño madero, i la superior hai dos agujerillos. En uno de esos se introduce una varilla como de 35 centímetros, que lleva embutida un cuña de palo mas frájil. Figura núm. 28. Sujetándose la pieza de los agujeros con los pies, cuando no hai otra persona que lo mantenga fijo, se da a la cuña un movimiento jiratorio rápido con la palma de las manos. Sobre unas hojas secas $u$ otra materia inflamable, cae un porción de aserrín caldeado, que, soplado, oportunamente, produce la combustión (Guevara 1911:621).

Otros silicofitolitos detectados poseen paredes gruesas y formas triangulares, aovadas o cuadrangulares. Estas células pueden proceder de tejidos parenquimáticos, presentes en epidermis de tallos y en las estructuras más duras de las hojas. Se registraron algunos asimilables a un conjunto de células, con las mismas características que las anteriormente descritas, en el hornillo de la pipa sin contexto. Este conjunto muestra similitudes, en el grosor de las paredes y tamaño de este conjunto de células, con las que se identificaron en la hoja de Nicotiana sp. y tallo de Peumus boldus (boldo) de la colección de referencia (Planella et al. 2009). Sin duda, estos resultados deben ser pasados a prueba a través de réplicas de las muestras y una comparación acuciosa que incluya mediciones más precisas. Pese a ello, no existe certeza acerca del modo de incorporación de estos silicofitolitos en el contenido de las pipas; su presencia bien puede responder a procesos de contaminación o propiamente haber sido consumido para fumar. Ambos taxa tiene asidero de consumo, los escritos mencionan que no se consumía tabaco puro en la pipas, se agregaban otras plantas con el fin de aromatizar u otorgarle otras propiedades al producto fumado (Guevara 1911; Guevara y Oyarzún 1912; Latcham 1936; Housse 1940; Cooper 1949). El boldo es nombrado explícitamente por Guevara: Desde tiempo inmemorial hasta la actualidad, nuestros naturales han mezclado la nicotina, con la trituración de tallos de algunas plantas, como maqui (Aristotelia chilensis), boldo (Boldo fragans) i varias otras (1911:618).

Por otra parte, los silicofitolitos bulliformes, frecuentes en las boquillas de ambas pipas, denotan la presencia de hojas de gramíneas y tal vez otras, sin poder determinar precisamente especies o familias
(Esau 1976). Aunque la identificación taxonómica es amplia, estos silicofitolitos corroboran el uso de material foliáceo en las pipas.

De la misma manera se reconocen otros silicofitolitos, descritos como tricomas y sus bases, relacionados a la presencia de hojas, pero también de tallos o frutos. En el caso de ambas pipas, algunos permanecen sin identificar con la excepción del tricoma correspondiente a la descripción "acicular articulado" proveniente del hornillo de la pipa del Rasgo 4-sector Casa 1, asimilable a los tricomas de la familia Solanaceae, de acuerdo a la colección de referencia confeccionada para la zona centro-sur de Chile (Planella et al. 2009). Esta familia se revela de gran interés en los contextos fumatorios, ya que el tabaco (Nicotiana spp.), la papa (Solanum tuberosum), el palqui (Cestrum, parqui) y el chamico (Datura stramonium), son mencionados como plantas fumadas o usadas por los mapuches (Guevara 1911; Joseph 1930; Munizaga 1960; Olivos 2004). A pesar de la elocuencia de este resultado, se reserva la confirmación de esta determinación taxonómica para las instancias en que se replicarán las muestras de la colección de referencia y se estudiarán más extensamente los rasgos de este tricoma articulado.

A modo de correlato, en la boquilla de la pipa del Rasgo 4, sector Casa 1 , se registraron 4 granos de almidones, uno de los cuales se atribuye a simil Nicotiana sp.. El hilo centrado, los brazos de la cruz de extinción rectos y finalmente el tamaño del grano, son los rasgos que permiten otorgarles esta probable asignación taxonómica. Si bien dichos granos de almidón son esperables de encontrar en el contenido de las pipas, por el evidente consumo documentado de tabaco, no poseen propiedades de preservación favorables en medios menos protegidos de la matriz de un sitio, como aquellas que presentan los silicofitolitos. En reforzamiento a lo anterior, se observa una clara diferencia entre el contenido de los hornillos y las boquillas, sustentada en la presencia de los almidones en éstas y su ausencia en los primeros. De haberse introducido por contaminación los granos de almidón, se encontrarían con similar frecuencia en ambos sectores de la pipa. La asociación clara de estos almidones a la boquilla indica de alguna manera que se dan procesos de conservación diferenciados en ambos contextos (boquilla y hornillo) inherentes al acto de fumar, ya que los almidones 
tenderían a ser destruidos en el hornillo por las altas temperaturas de la combustión directa (Rafferty 2006; Muñoz y Peña 2009). La confirmación del uso de tabaco en estas pipas, sería de suma importancia para la comprensión de los complejos fumatorios de la región sur de Chile, ya que sólo se dispone referencias en las fuentes escritas.

Otro taxon probablemente pesquisado por la presencia de almidón en la pipa del Rasgo 4-sector Casa 1 , es Solanum spp. ${ }^{5}$. El hilo excéntrico, los lóbulos asimétricos y la forma del grano han permitido proponer el uso de esta planta. Esta determinación resulta interesante, considerando los registros del consumo de hojas de papa a modo de sucedáneo del tabaco (Joseph 1930). Igualmente, la presencia combinada, por cierto en estado de verificación, de tabaco y papa en una misma pipa, revela aspectos secuenciales o de modalidades de uso. Se debe tomar en cuenta que los microfósiles recuperados en un artefacto arqueológico refieren un promedio de uso, independientemente del orden o frecuencia en que sucedieron los episodios de consumo (Babot com. pers.).

Finalmente, otra vía de aproximación al problema de los contenidos de las pipas, es la interpretación de los daños presentes en los granos de almidón, constituye otra fuente de información que permite inferir los tratamientos infligidos a los materiales fumados. En esta perspectiva, una primera observación es la presencia diferencial de los almidones en boquillas y hornillos; la única excepción se registra en el hornillo de la pipa sin contexto, que cuenta con un almidón con muy baja birrefringencia. En segundo lugar, se aprecian diferencias en los daños que muestran los almidones de una pipa y otra. Mientras, la pipa del Rasgo 4 , sector Casa 1 , presentan daños relacionados a la deshidratación, el tostado o carbonización (i.e. a alteraciones del hilo y birrefringencia, fisuras, visibilidad aminorada), en la pipa sin contexto los

5 Puede corresponder a papa silvestre (Solanum maglia) como también a cultivada (Solanum tuberosum), hay antecedentes del uso de ambos para fumar (Serrano 1934; Housse 1940). A la luz de los datos etnográficos consultados sobre los grupos Tehuelches y el uso de las pipas tipo monitor, pueden considerarse las especies Myrceugenia pitra y Berberis buxifolia, ya que estas plantas aromáticas remplazaban o se mezclaban con el tabaco cuando este escaseaba, esta modalidad también era perpetrada por los mapuches (Martinic 1991). almidones presentan huellas de desecamiento, molienda y, en menor medida, tostado o carbonizado (i.e. relieve modificado, alteraciones de la birrefringencia y visibilidad del hilo) (Babot 2003, 2006). Estas diferencias evidencian parte de los tratamientos aplicados a los elementos fumados en las pipas. Después de todo, la conservación de un ejemplar de grano de almidón responde a procesos multifactoriales y, por ende, el contenido rescatado en un pipa no refleja la totalidad de los episodios de consumo, sino la ínfima parte de lo que se conservó hasta el momento de la extracción de microfósiles.

\section{CONCLUSIÓN}

Los elementos más frecuentemente detectados en ambas pipas, remiten a la presencia de especies relacionadas a la producción de fuego, mayoritariamente gramíneas, pero que en algunos casos podrían corresponder a la subfamilia Bambusoideae, género Chusquea (C. culeou, C. cumingii). En segundo lugar, desde el punto de vista del origen anatómico de los tejidos vegetales involucrados, la presencia de silicofitolitos denominados células bulliformes y parenquimáticas indica el uso de hojas y tallos. En tercer lugar, se observó que los daños exhibidos por los almidones, permitiendo inferir el tostado, la carbonización y la molienda de los vegetales consumidos en el acto de fumar.

Con un grado menor de certeza, debido a la reiteración de estructuras celulares recuperadas, se ha considerado la presencia de una planta con propiedades psicotrópicas (Nicotiana sp,) o aromáticas (Peumus boldus). Por otra parte, los almidones podrían confirmar la incorporación de Nicotiana sp. y de Solanum spp., usado este último como sucedáneo del tabaco.

En el estado actual de este estudio sobre las pipas del sitio Villa JMC-1 Labranza, es posible concluir con mayor certeza acerca de la participación de Poaceae o gramíneas, de las partes anatómicas usadas y de las modalidades de tratamiento aplicado a los materiales vegetales consumidos en las pipas. En lo que respecta la identificación taxonómica definitiva de los microfósiles rescatados se prefiere esperar los resultados de nuevas replicas de la colección de referencia, antes de admitirlas como aporte definitivo a la investigación. 


\section{AGRADECIMIENTOS}

Al profesor Eugenio Aspillaga, por facilitar el uso de las instalaciones del Laboratorio de Antropología Física del Depto. de Antropología, Universidad de Chile. Además agradecemos la acogida de Álvaro Villagrán y Roxana Seguel en el Laboratorio de Análisis del Centro Nacional de Conservación y Restauración. Extendemos nuestros agradecimientos a Hermann Niemeyer del Dept. de Ciencias Ecológicas, Facultad de Ciencias, Universidad de Chile quien facilitó la infraestructura del laboratorio de su unidad academica.

\section{BILBIOGRAFÍA}

ARMSTRONG H. y BRASIER M. 2005. Microfossils. Blackwell Publishing, Oxford.

BABOT, M.P. 2003. Starch grain damage as an indicator of food processing. En: Phytolith and starch research in the Australian-Pacific-Asian regions: the state of the art, editado por D. Hart y L. Wallis, pp: 69-81. Pandamus Books for the Centre for Archaeological Research. Canberra.

2004. Tecnología y utilización de artefactos de molienda en el Noroeste prehispánico. Tesis doctoral no publicada. Facultad de Ciencias Naturales e IML, Universidad Nacional de Tucumán. San Miguel de Tucumán.

2006. Box 4.4: Damage on starch from processing Andean food plants. En: Ancient Starch Research, editado por R. Torrence y H. Barton, pp. 66-67. Left Coast Press. California.

2007. Granos de almidón en contextos arqueológicos: posibilidades y perspectivas a partir de casos del noroeste argentino. En: Paleoetnobotánica del cono sur: estudios de casos y propuestas metodológicas, editado por B. Marconetto, N. Oliszewski y M. P. Babot, pp. 95-125. Ferreyra Editor. Córdoba.

BARTON, H., R. TORRENCE y R. FULLAGAR. 1998. Clues to stone tools function re-examined: comparing starch grain frequencies on used and unused obsidian artifacts. Journal of Archaeological Science 25: 1231-1238.

BERTOLDI DE POMAR, H. 1972 Ópalo organógeno en sedimentos superficiales de la llanura Santafesina. Amghinnianna IX (3):265-279.

BRIUER, F. 1976. New clues to stone tool functions: plants and animal residues. American Antiquity 41(4):478-484.

CAPPARELLI, A., M. POCHETTINO; A. DIEGO y R. ITURRIZA. 2006. Differences between written and archaeological record: the case of plant micro remains recovered at a Northwestern Argentinean Pipe. En: Proceedings of the IV International Congress of Ethnobotany, editado por Z. Fusun Ertu, pp: 397-406. Zero Prod. Ltd. Estambul.

COIL, J., A. KORSTANJE, S. ARCHER y C. HARSTOF. 2003. Laboratory goals and considerations for multiple microfossil extraction in archaeology. Journal of Archaeological Science 30: 991-1008.

COOPER, J. 1949. Stimulants and narcotics. En: Handbook of South American Indians, editado por J. Stewart, 5:525-558. Smithsonian Institution Bureau of American Archaeology Bulletin 143. New York.

ESAU, K. 1976. Anatomía vegetal. Editorial Omega, Barcelona. FÖERSTER, R. 1995. Introducción a la religiosidad mapuche. Editorial Universitaria. Santiago.

FÖERSTER, R. y H. GUNDEMANN. 1996. Religiosidad mapuche contemporánea: elementos introductorios. En: Etnografía, sociedades indígenas contemporáneas y su ideología, editado por J. Hidalgo, V. Schiappacasse, H. Niemeyer, C. Aldunate y P. Meges, pp: 189-240. Editorial Andrés Bello. Santiago.

FULLAGAR, R., FURBY, J. y B. HARDY. 1996. Residues on stone artifacts: state of a scientific art. Antiquity 70:740-745.

GUEVARA, T. 1911 Folklore Araucano: refranes, cuentos, cantos, procedimientos industriales, costumbres prehispanas. Imprenta Cervantes. Santiago.

GUEVARA, T. y A. OYARZÚN. 1912. El tabaco y las pipas prehistóricas de Chile. En: Actas del XVII Congreso Internacional de Americanistas, pp. 414-437. Imprenta y Casa Editora Juan A. Alsina. Buenos Aires

HOUSSE, E. 1940. Una epopeya india. Editorial Zig-Zig. Santiago. JOSEPH, C. 1930 Antigüedades de Araucanía. Revista Universitaria Católica de Chile XV (9): 1171-1235

ICPN WORKING GROUP: MADELLA, M., A. ALEXANDRE y T. BALL. 2005. International code for phytolith nomenclature 1.0. Annals of Botany 96(2):253-260.

KEALHOFER, R. TORRENCE y R. FULLAGAR. 1999. Integrating phytoliths within use-wear/residues studies of stone tools. Journal of Archaeological Science 26: 527-546.

KORSTANJE, M. A. 2005. La organización del trabajo en torno a la producción de alimentos en sociedades agropastoriles formativas (Pcia. de Catamarca, Rep. Argentina). Tesis doctoral no publicada. Facultad de Ciencias Naturales e IML, Universidad Nacional de Tucumán. San Miguel de Tucumán. MS.

2006. Box 8.5: Designing a protocol for the simultaneous recovery of multiple microfossils. En: Ancient starch research, editado por R. Torrence y H. Barton, pp: 162-163. Left Coast Press. California. 
2009. Microfósiles y agricultura prehispánica: primeros resultados de un análisis múltiple en el Noroeste Argentino. En: Fitolitos: estado actual de sus conocimientos en América del Sur, editado por A. Zucol, M. Osterrieth y M. Brea. Universidad Nacional de Mar del Plata. Mar del Plata.

KORSTANJE, M. A. y M. P. BABOT. 2007. A microfossil characterization from South Andean economic plants. En: Plants, people and places: recent studies in phytholithic analysis, editado por M. Madela y D, Zurro, pp. 41-72. Oxbow Books. Cambridge.

LATCHAM, R.E. 1936. La agricultura precolombiana en Chile y los países vecinos. Ediciones de la Universidad de Chile. Santiago.

LOY, T. 1994. Methods and analysis of starch residues of prehistoric stones tools. En: Tropical Archaeobotany: application and new development, editado por J. Hater, pp: 86-114. Routledge. New York.

LOY, T. y R. FULLAGAR. 2006. Box 9.8. Residue extraction. En: Ancient Starch Research, editado por R. Torrence y H. Barton, pp. 197-198. Left Coast Press. California.

MARTINIC M. 1991. El hábito de fumar entre los Aonikenk. Anales del Instituto de la Patagonia, serie Ciencias Sociales. 20: 19-28.

MERA, R. y D. MUNITA. 2008. Informe Ejecutivo Salvataje Sitio Villa JMC-01, Labranza, Provincia de Cautín, Región de La Araucanía. MS.

MU Phytholith Database. http://database.coas.missouri.edu/ $\mathrm{fmi} / \mathrm{xsl} / \mathrm{phytos} /$ searchxsl.

MUNIZAGA, C. 1960 Nota sobre el uso de Miyaya (Datura stramonium) por los araucanos chilenos actuales en el tratamiento de trastornos mentales. Revista Universitaria XLIV-XLV (23):43-45.

MUÑOZ, O. y R. PEÑA. 2009 Investigaciones recientes en la determinación de residuos de pipas arqueológicas de Chile central. Boletín del Museo Nacional de Historia Natural 58: 83-89.

OLIVOS, C.G. 2004. Plantas psicoactivas de eficacia simbólica: indagaciones en la herbolaria mapuche. Chungara 36(2):997-114.

PEARSALL, D.1989 Paleoethobotany: a handbook of procedure. Academic Press. San Diego.
PEARSALL, D. y D. PIPERNO. 1993. Current research in phytolith analysis: applications in archaeology and paleoecology. University of Pennsylvania Museum of Archaeology and Anthropology. Philadelphia.

PIPERNO, D. 1988. Phytolith Analysis: an archaeological and geological perspective. Academic Press. London. 2006. Phytoliths: a comprehensive guide for archaeologists and paleoecologists. AltaMira Press. Maryland.

PIPERNO, D. e I. HOLST. 1998. The presence of starch grains on prehistoric stone tools from the humid neotropics: indications of early tuber use and agriculture in Panama. Journal of Archaeological Science 25: 765-776.

PIPERNO, D. y PEARSALL, D. 1998. The silica bodies of tropical American grasses: morphology, taxonomy, and implications for grass systematics and fossil phytolith identification. Smithsonian Contribution to Botany 85:1-40

PLANELLA, M.T., L. QUIROZ, C. BELMAR y V. MCROSTIE. 2009. Explorando el bosque esclerófilo: fitolitos y almidones de la flora nativa de Chile central. Resúmenes del XVIII Congreso Nacional de Arqueología Chile, pp.201-203. Gráfica LOM. Valparaíso.

PLANELLA, M.T., G. SANTANDER y V. MCROSTIE. 2011. Estudio morfotecnológico y análisis de microfósiles en piedras tacitas de Chile central. En: Entre las muchas historias entre las plantas y la gente: alcances y perspectivas de los estudios arqueobotánicos en América Latina, editado por S. Rojas-Mora y C. Belmar. British Archaeological Reports. En prensa.

RAFFERTY, S.M. 2006. Evidence of early tobacco in Northeastern North America. Journal of Archaeological Science 33:453-458.

REICHERT, E.T. 1913. The differentiation and specificity of starches in relation to Genera, Species, Etc. Carnegie Institution of Washington. Washington D.C.

SERRANO, A. 1934. El uso del tabaco y vegetales narcotizantes entre los indígenas de América. Revista geográfica Americana II (15):415-425.

ZUCOL, A.F. y PASSEGGI 2008. Análisis fitolíticos: metodologías básicas y su aplicación a los estudios paleocológicos. Guía para el desarrollo de la cursada. MS. 
